\title{
Fatores de risco físicos e organizacionais associados a distúrbios osteomusculares relacionados ao trabalho na indústria têxtil
}

\author{
Physical and organisational risk factors associated to work-related \\ musculoskeletal disorders in textile industry
}

\author{
Adriana Cristina de Souza Melzer
}

Fisioterapeuta Ms.; doutoranda em Saúde Coletiva na Faculdade de Ciências Médicas, Depto. de Medicina Preventiva e Social da Unicamp (Universidade Estadual de Campinas, Campinas, SP)

ENDEREÇO PARA

CORRESPONDÊNCIA

Adriana C. S. Melzer

R. Mons. Fergo O.C. Dauntre 768

13045-685 Campinas SP

e-mail:

adrianamelzer@hotmail.com

Estudo financiado pela Capes

Apoio financeiro: Fapesp

(Processo n 03/12099-0)
ApresentaÇÃO set. 2006

ACEITO PARA PUBLICAÇÃO dez. 2007
Resumo: Distúrbios osteomusculares relacionados ao trabalho (DORT) atingem trabalhadores de diversas ocupações e constituem um dos maiores problemas de saúde em muitos países. Este estudo foi realizado em dois setores de fiação de uma mesma indústria têxtil, sendo um deles tecnologicamente mais moderno. Os objetivos foram identificar os fatores de risco físicos e organizacionais associados aos DORT nos dois setores e determinar a prevalência de sintomas de dor entre esses trabalhadores. Tendo como referencial a análise ergonômica, foram observadas as atividades de trabalho de 12 indivíduos; e 50 trabalhadores responderam a um questionário. Os fatores de risco físico identificados foram os seguintes: posturas inadequadas de ombro, trabalho na posição em pé, repetitividade de movimentos e aspectos ambientais desfavoráveis. Os fatores organizacionais identificados foram: ritmo intenso de trabalho, fragmentação e invariabilidade das tarefas, inexistência de pausas e impossibilidade de comunicação com os colegas. Foi encontrada prevalência de 60\% e 76\% de dor nos dois setores estudados, respectivamente. As conclusões mostram que investimentos em tecnologia que não acompanham mudanças na organização e nas condições de trabalho resultam na manutenção ou no agravamento das situações de risco reconhecidamente associadas aos DORT.

DESCRITOREs: Engenharia humana; Indústria têxtil; Fatores de risco; Transtornos traumáticos cumulativos

ABSTRACT: Work-related musculoskeletal disorders (WRMD) affect workers in several occupations and are one of the major health problems in many countries. This study was developed in two sectors of a textile industry, one of them being technologically more advanced. The objectives were to identify physical and organizational risk factors associated to WRMD in the two sectors and estimate prevalence of pain among these workers. The workplace and working activity of 12 individuals were observed on ergonomic grounds, and 50 workers answered a questionnaire. The following physical risk factors were identified: awkward shoulder postures, standing work position, repetitive movements, and unfavourable environmental features. Main organizational risk factors identified were: intense work pace, uneven and repetitive tasks, absence of pauses and impossibility of communication with peers. Pain symptoms prevalence of $60 \%$ and $76 \%$ was found in the two sectors. Conclusion shows that investments in technology that do not follow changes in work condition and organization may result in maintenance or aggravation of risk situations associated to WRMD.

KEY WORDs: Cumulative trauma disorders; Human engineering; Risk factors; Textile industry 


\section{INTRODUÇÃO}

Distúrbios osteomusculares relacionados ao trabalho (DORT) destacam-se como um dos maiores problemas de saúde pública dos dias atuais, gerando impactos econômicos e sociais em diversos países.

Registrados inicialmente no Japão em 1958, nas ocupações de operadores de caixa registradora, perfuradores de cartão e datilógrafos, os DORT invadiram progressivamente as mais diversas indústrias e ocupações até, atualmente, não respeitarem fronteiras entre as categorias profissionais ${ }^{1-3}$. Estudos epidemiológicos realizados em diferentes países identificaram casos de DORT em montadoras, confecções, indústrias de construção, indústrias fabricantes de embalagens, tesouras, moldes e cabines de caminhão e entre as ocupações de açougueiros, laminadores, telegrafistas, digitadores, enfermeiros e taxistas, entre outras ${ }^{4-12}$.

Embora a causa dos DORT seja uma questão não completamente elucidada, prevalece um consenso quanto à natureza multifatorial, em que diversos fatores em interação contribuem para o desenvolvimento do distúrbio. Estudos epidemiológicos têm mostrado a relação entre fatores de risco físicos como repetitividade, força muscular e posturas inadequadas, extremas e estáticas, e distúrbios osteomusculares $^{13-16}$. Outros estudos identificam associação entre os DORT e fatores de risco organizacionais e psicossociais, como períodos prolongados de trabalho, altas demandas de trabalho, pressão por tempo e ambiente social de trabalho ${ }^{12,17-20}$.

Os objetivos deste estudo foram identificar os fatores de risco físicos e organizacionais ou psicossociais associados ao desenvolvimento de DORT em dois setores de fiação de uma indústria têxtil e determinar a prevalência de sintomas de dor e/ou desconforto osteomuscular.

\section{METODOLOGIA}

Foi realizada uma análise descritiva e comparativa de dois setores de fiação e de postos de trabalho de fiandeiros de uma mesma indústria têxtil de grande porte localizada em Fortaleza, Ceará, tendo como referencial a análise ergonômica do trabalho.

Os setores de fiação estão localizados entre os setores de preparação do algodão e o setor de acabamento e têm como função produzir "espulas" de fio de algodão que posteriormente seguem para a "conicalagem", onde são formados os cones de fio. Os dois setores foram escolhidos porque, seguindo o processo de modernização iniciado pela empresa em 1997, um deles é dotado de equipamentos mais modernos, o que é traduzido tanto em termos de diferenças na estrutura do maquinário, como altura mais elevada e maior número de fusos, quanto em termos tecnológicos, apresentando um acréscimo que torna automatizada uma das atividades relacionadas à fiação. Por esse motivo e para efeito de nomenclatura, os dois setores estudados foram aqui identificados como "setor modernizado" e "setor antigo".

O fiandeiro é responsável pela inspeção consecutiva dos filatórios (máquinas de fiação que contêm fusos sobre os quais se forma a "espula" de algodão), durante a qual executa duas atividades principais: a emenda dos fios quebrados e a troca das "maçarocas". Na atividade de emenda dos fios, os fiandeiros freiam a máquina com um dos joelhos, enquanto com as duas mãos retiram a espula do fuso e emendam o fio rompido. Na atividade de troca da "maçaroca", os fiandeiros retiram a "maçaroca" vazia, alcançam uma cheia em cima do filatório e a encaixam na máquina para rodar o processo.

\section{Observação direta das atividades de trabalho}

Em cada setor de fiação, foram observadas as atividades de trabalho de 6 fiandeiros, sendo 2 em cada turno de trabalho (manhã, tarde e noite), totalizando 12 observações. Cada fiandeiro foi observado durante $30 \mathrm{mi}$ - nutos, em dias da semana alternados, abrangendo-se todos os turnos de trabalho.

As observações basearam-se em um Roteiro de Observação adaptado de McAtamney e Corlett ${ }^{21}$ e orientado para a investigação de aspectos gerais do trabalho, como jornada de trabalho, turno, pausas, rodízios, características do maquinário, fatores ambientais e EPIs (equipamentos de proteção individual); aspectos específicos da atividade do fiandeiro como tempo total de trabalho, descrição, duração e repetitividade das atividades principais e auxiliares, avaliação das posturas e movimentos por segmento corporal; e avaliação de aspectos organizacionais como ritmo, variedade no trabalho e comunicação entre os trabalhadores.

\section{Entrevista}

Um total de 50 fiandeiros, 25 em cada setor de fiação, foram escolhidos aleatoriamente e responderam a um questionário. Utilizou-se um questionário misto, constituído por perguntas abertas e fechadas, com informações sobre dados sociodemográficos, tempo de trabalho na indústria e na função de fiandeiro, sistema de trabalho, controle sobre o ritmo, repetitividade, pausas, posturas, altura da máquina, ambiente físico geral, aspectos relacionados à saúde e presença de dor e/ou desconforto em alguma parte do corpo.

O perfil sociodemográfico dos trabalhadores entrevistados foi o seguinte: 29 mulheres e 21 homens, média de idade de $32,2 \pm 8,0$ anos, altura média de $1,62 \pm 0,10 \mathrm{~m}$, tempo médio de trabalho na indústria 6 anos e meio e, na função de fiandeiro, média de 5 anos e um mês.

Os dados obtidos a partir dos questionários foram analisados com auxílio do programa estatístico SPSS $\mathbb{R}$ para Windows.

\section{RESULTADOS}

As características gerais dos dois setores de fiação e aspectos específicos 
Quadro 1 Características dos setores de fiação e da atividade de fiandeiro

\begin{tabular}{|c|c|c|}
\hline $\begin{array}{l}\text { Setor de fiação } \\
\text { Características }\end{array}$ & Setor modernizado & Setor antigo \\
\hline Número de fiandeiros & $\begin{array}{c}58 \text { (33 homens, } 25 \\
\text { mulheres) }\end{array}$ & $\begin{array}{c}69 \text { (19 homens, } 50 \\
\text { mulheres) }\end{array}$ \\
\hline
\end{tabular}

Sistema de turnos Turnos fixos, diurno, vespertino e noturno

Extensão da jornada de trabalho

48 horas semanais

\begin{tabular}{|lc|c|}
\hline Pausas & \multicolumn{2}{c|}{ Não ocorrem pausas curtas } \\
\hline Ruído & $92 \mathrm{a} 93 \mathrm{~dB}$ & $94 \mathrm{~dB}$ \\
\hline Iluminação (média) & $158,25 \mathrm{lux}$ & $101,46 \mathrm{lux}$ \\
\hline Poeira de algodão & $0,52 \mathrm{mg} / \mathrm{m}^{3}$ & $0,67 \mathrm{mg} / \mathrm{m}^{3}$ \\
\hline IBUTG médio & $26,02{ }^{\circ} \mathrm{C}$ & $25,37{ }^{\circ} \mathrm{C}$ \\
\hline
\end{tabular}

\begin{tabular}{|c|c|c|}
\hline Organização do trabalho & \multicolumn{2}{|c|}{$\begin{array}{l}\text { Trabalho individualizado; divisão entre } \\
\text { concepção e execução }\end{array}$} \\
\hline odução & \multicolumn{2}{|c|}{$\begin{array}{c}\text { Determinado pela velocidade de rotação dos } \\
\text { fusos (13.000 rpm) e influenciado pela } \\
\text { qualidade do algodão }\end{array}$} \\
\hline $\begin{array}{l}\text { Comunicação entre } \\
\text { trabalhadores }\end{array}$ & \multicolumn{2}{|c|}{ Limitada pelo ruído e ritmo de trabalho } \\
\hline $\begin{array}{l}\text { Altura máxima da máquina } \\
\text { (filatório) }\end{array}$ & $2,20 \mathrm{~m}$ & $2,10 \mathrm{~m}$ \\
\hline $\begin{array}{l}\text { Duração média / Repetição } \\
\text { média da atividade de } \\
\text { emenda dos fios }\end{array}$ & $\begin{array}{l}4 \text { a } 6 \text { seg./ } 1.028 \\
\text { vezes ao dia }\end{array}$ & $\begin{array}{l}5 \text { a } 7 \text { seg./ } 1.171 \\
\text { vezes ao dia }\end{array}$ \\
\hline $\begin{array}{l}\text { Duração média / Repetição } \\
\text { média da atividade de } \\
\text { troca da maçaroca }\end{array}$ & $\begin{array}{c}6 \text { a } 9 \text { seg./ } 312 \text { vezes } \\
\text { ao dia }\end{array}$ & $\begin{array}{c}7 \text { a } 9 \text { seg./ } 205 \text { vezes } \\
\text { ao dia }\end{array}$ \\
\hline
\end{tabular}

Em pé (não estática) durante toda a jornada; movimentos de elevação de ombro acima da cabeça; movimentos de flexão, extensão,

Posturas e movimentos desvio radial e ulnar e circundução de punho

EPI = equipamento de proteção individual; IBUTG = Índice de Bulbo Úmido Termômetro de Globo (avalia a exposição ocupacional ao calor)

da atividade de trabalho de fiandeiro podem ser vistos no Quadro 1.

Os setores de fiação analisados assemelham-se em alguns aspectos gerais do trabalho, como sistema de turnos, extensão da jornada e ausência de pausas curtas. Com relação aos aspectos ambientais, embora os dados fornecidos pelo setor de segurança no trabalho revelem algumas variações, pode-se considerar que ambos os setores apresentam níveis elevados de ruído, justificando a utilização de protetores auriculares por todos os trabalhadores. As informações sobre iluminação - aspecto importante na atividade de emenda dos fios - não refletem a realidade, uma vez que há locais, em ambos os setores, com pouca iluminação, embora a dispo- estratégias que se enquadram no movimento da qualidade total com destaque para os "cinco S".

O ritmo de trabalho, determinado pela velocidade de rotação dos fusos (13.000 rpm), é elevado nos dois setores. A qualidade do algodão interfere com o ritmo de trabalho, na medida em que determina o grau de resistência e quebra do fio, ou seja, quanto pior a qualidade do algodão, maior a quebra dos fios e mais intenso o ritmo.

No setor modernizado, cada fiandeiro é responsável pelo monitoramento de cerca de 3.000 fusos enquanto no setor antigo, cada um é responsável por cerca de 2.600 fusos.

Em ambos os setores, as atividades principais do fiandeiro (emenda dos fios e troca da "maçaroca") caracterizam-se como atividades de curta duração (de 4 a 9 segundos) e alta repetitividade (mais de 1.000 vezes ao dia para atividade de emenda dos fios e entre 200 e 300 vezes para troca da "maçaroca"). Durante toda a jornada de trabalho, os fiandeiros permanecem em pé, monitorando as máquinas de fiação e alternando posturas de elevação do braço acima da altura da cabeça (quando alcançam a "maçaroca" no topo da máquina) e movimentos repetidos de punho.

As opiniões dos trabalhadores quanto aos aspectos relacionados a fatores físicos gerais do trabalho e os ligados à organização do trabalho são apresentadas na Tabela 1, com base nas respostas ao questionário.

Os dados apresentados na Tabela 1 foram comparados estatisticamente pelo teste de $\chi 2$. As diferenças que se mostraram estatisticamente significativas são as relativas ao controle sobre o ritmo de trabalho $(\chi 2=10,784$; $p=0,001$ ) è repetitividade do trabalho $(\chi 2=4,348 ; p=0,037)$. A maioria dos trabalhadores do setor antigo referiu ter controle sobre o ritmo de trabalho. O trabalho foi considerado repetitivo pela maioria dos trabalhadores dos dois setores, com maior percentual no setor antigo. 
Tabela 1 Distribuição percentual de trabalhadores segundo as respostas "sim" às perguntas sobre fatores físicos e organizacionais do trabalho $(\mathrm{N}=50)$

\begin{tabular}{|c|c|c|}
\hline Características & $\begin{array}{l}\text { Modernizada } \\
\text { SIM \% (n) }\end{array}$ & $\begin{array}{c}\text { Antiga } \\
\operatorname{SIM} \%(n)\end{array}$ \\
\hline Considera a iluminação adequada & $84(21)$ & $88(22)$ \\
\hline Considera o nível de ruído tolerável & $36(9)$ & $32(8)$ \\
\hline Considera a temperatura agradável & $16(4)$ & $20(5)$ \\
\hline Controla o ritmo de trabalho & $44(11)$ & $88(22)$ \\
\hline Considera o trabalho repetitivo & $84(21)$ & $100(25)$ \\
\hline Participa nas decisões & 0 & 0 \\
\hline Tem bom relacionamento com colegas & $100(25)$ & $96(24)$ \\
\hline Está satisfeito com a chefia & $92(23)$ & $92(23)$ \\
\hline Acredita em promoção & $40(10)$ & $44(11)$ \\
\hline
\end{tabular}

Uma das perguntas mais importantes do questionário era "Você sente alguma dor ou desconforto em alguma parte do corpo?". Entre os entrevistados, $60 \%$ (15) do setor modernizado e $76 \%$ (19) do setor antigo admitiram sentir alguma dor e/ou desconforto. Não foram encontradas associações significativas entre dor/desconforto e setor $(\chi 2=0,082 ; p>0,05)$, dor/desconforto e turnos $(\chi 2=2,665 ; p>0,05)$, dor/desconforto e sexo $(\chi 2=1,961$; $p>0,05)$, dor/desconforto e faixa etária $(\chi 2=0,999 ; p>0,05)$, dor/ desconforto e tempo de trabalho na indústria $(\chi 2=7,862 ; p>0,05)$, nem quanto ao tempo de trabalho como fiandeiro $(\chi 2=4,711 ; p>0,05)$. Os locais do corpo mais freqüentemente referidos como dolorosos foram: perna direita $(25,3 \%)$, perna esquerda $(24 \%)$, coluna lombar $(10,7 \%)$, ombro direito (8\%), coluna torácica $(5,4 \%)$, coluna cervical (4\%), ombro esquerdo (4\%) e joelho esquerdo $(4 \%)$. As respostas mais freqüentes à pergunta "o que você acha que causa essa dor" foram: "dias muito puxados" (32,3\%), "ficar muito em pé" $(17,6 \%)$, "atividade" $(17,6 \%)$, "frear a máquina" $(8,8 \%)$, "máquinas muito altas" (5,88\%) e outras como "andar muito", "movimentos" e "peso em uma perna".

\section{DISCUSSÃO}

A expectativa inicial era de que os investimentos da empresa na modernização de um de seus setores de fiação, incluindo a adoção de maquinário aperfeiçoado e semi-automatizado e a reestruturação do ambiente físico, exerceriam efeitos favoráveis à empresa e aos trabalhadores, agilizando a produção e garantindo melhores condições de trabalho. No entanto, o estudo revelou que o incremento da produtividade, garantido pelo maior número de fusos por máquina e pela manutenção do alto ritmo de rotação do filatório, assim como o ganho de tempo possibilitado pela automatização de parte do processo, não vieram acompanhados de condições adequadas de trabalho, pelo contrário, acabaram por perpetuar as situações de risco.

Por conseguinte, em vez de se encontrarem diferenças organizacionais e em termos de sobrecarga física no setor modernizado, bem como um menor índice de queixas osteomusculares, encontraram-se semelhanças e aspectos críticos desfavoráveis, além de alta prevalência de dor, em ambos os setores.

\section{Fatores de risco físicos}

Os principais fatores de risco físicos associados aos DORT identificados foram repetitividade de movimentos, trabalho na posição em pé durante toda a jornada e posturas extremas de ombro. Esses fatores de risco estão entre os mais citados na literatura ${ }^{22-25}$.

O trabalho dos fiandeiros pode ser considerado como altamente repe- titivo ${ }^{4} \mathrm{em}$ ambos os setores de fiação. Essa característica torna-se evidente tanto na observação direta das atividades quanto na opinião dos trabalhadores que, em sua maioria, consideram o trabalho repetitivo.

As atividades são executadas na posição em pé, sem possibilidades de variação de postura. Como conseqüência, o trabalhador pode manifestar algias, fadiga e distúrbios osteomusculares devido à carga estática exigida para manter a posição de pés, joelhos, quadris e coluna ${ }^{26,27}$. Nos depoimentos dos trabalhadores, as pernas foram o segmento mais mencionado para a ocorrência de dor ou desconforto e "ficar muito em pé" foi a segunda maior causa de dor apontada pelos entrevistados, comprovando o potencial de risco que esse padrão postural acarreta.

Os fiandeiros também executam movimentos repetidos de elevação dos braços acima da altura da cabeça, como na atividade de troca da maçaroca, o que, como se sabe, é comprovadamente lesivo para as estruturas do ombro. Esse aspecto também reflete a inadequação das máquinas às características antropométricas da população, situação que se agrava ainda mais no setor modernizado, em que os filatórios foram elevados com vistas à maior produtividade.

Além destes, aspectos relacionados ao conforto proporcionado pelo ambiente de trabalho também podem ser incluídos entre os fatores de risco físicos. Ruído excessivo, temperatura elevada e iluminação inadequada em alguns locais podem ser determinantes na adoção de determinadas posturas e podem contribuir para um aumento dos níveis de estresse no trabalho, como referem outros autores ${ }^{28}$.

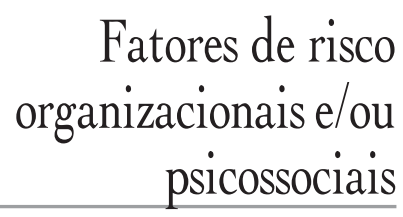

Entre os fatores de risco organizacionais ou psicossociais do trabalho foram identificados: ritmo intenso de 
trabalho, fragmentação e invariabilidade das tarefas, inexistência de pausas e impossibilidade de comunicação com os colegas. Esses dados são condizentes com estudos que identificam associação entre fatores organizacionais e/ou psicossociais e DORT ${ }^{12,18,19,29}$. O trabalho em ambos os setores de fiação orienta-se segundo os padrões do taylorismo/fordismo. As atividades dos fiandeiros são fragmentadas, invariáveis, executadas em ritmo intenso e sem pausas.

$\mathrm{O}$ aumento da produtividade no setor modernizado decorreu da utilização de um menor número de máquinas, capazes de produzir a mesma quantidade de fio de algodão com uma significativa economia de tempo. Aliado a isso, os fiandeiros do setor modernizado passaram a ser responsáveis pelo patrulhamento de um maior número de fusos (3.000) em relação aos do setor antigo (2.640). Dessa forma, pode-se dizer que o processo de modernização traduziu-se na intensificação do trabalho dos fiandeiros que, em nome da produtividade e do menor tempo, foram sobrecarregados em suas funções.

Esse quadro acompanha o panorama geral das novas formas de gestão e controle do trabalho que emergem no Brasil a partir da década de 1980 e que, baseadas no aumento da produtividade, na eficiência, na qualidade e em novas formas de gestão, representam uma ampliação da exploração da força de trabalho, seja pela maisvalia relativa (inovação tecnológica) ou mais-valia absoluta (ampliação do ritmo de trabalho) ${ }^{30}$. Para Dejours ${ }^{31}$, trabalhadores submetidos a esse tipo de organização do trabalho vivenciam uma situação impregnada pelo risco de não acompanhar o ritmo de trabalho, gerando um estado de tensão e ansiedade. A intensificação do trabalho, quando não se buscam formas de proteger os trabalhadores da sobrecarga, da tensão e da fadiga, pode superar as possibilidades psicobiológicas do trabalhador ${ }^{32}$, favorecendo o desenvolvimento do distúrbio osteomuscular, entre outros.

Vários estudos identificam a relação entre trabalho em ritmo elevado e distúrbios osteomusculares. Comparando duas indústrias produtoras de vidro em diferentes fases de incorporação tecnológica, Queiroz e Maciel ${ }^{33}$ encontraram um maior número de queixas de dores musculares na indústria em que o ritmo de trabalho é mais elevado. No estudo de Fassa et al. ${ }^{34}$, dores nas costas, cansaço e irritação foram relatados por trabalhadores de um dos setores de uma indústria de celulose e papel como os principais problemas de saúde. Nesse setor, trabalhar em grande velocidade (referida por $56,5 \%$ dos trabalhadores) foi considerada uma das principais cargas de trabalho relacionadas à atividade. Ghisleni e Merlo ${ }^{35}$ entrevistaram trabaIhadores portadores de DORT em Porto Alegre e apontaram o ritmo de trabalho elevado como um dos principais fatores organizacionais responsáveis pelo desenvolvimento do distúrbio.

Ainda no contexto de transformação iniciado pela empresa, vale ressaltar que investidas em programas de qualidade como os "cinco S" pouco significaram para os trabalhadores da fiação que, em essência, não vêem em absoluto alteradas suas condições de trabalho. Isso é condizente com estudos que mostram que as melhorias nas condições de trabalho efetuadas pelas empresas brasileiras estão restritas a questões de higiene, limpeza e organização dos locais de trabalho, deixando de lado pontos cruciais para a saúde dos trabalhadores, como o ritmo acelerado de trabalho ${ }^{36}$.

Outro aspecto revelador da organização social do trabalho e próprio do modelo taylorista de organização do trabalho presente nos setores de fiação é a restrição da comunicação entre os trabalhadores $^{37}$. A restrição da comunicação entre colegas de trabalho segue uma das prerrogativas de Taylor, segundo a qual o trabalho bem planejado prescinde da comunicação entre trabalhadores. Todavia, como defendem alguns autores, quanto pior a qualidade da comunicação e as relações de suporte entre os trabalhadores, maiores os riscos de desenvolvimento de DORT ${ }^{18,38}$.

\section{CONCLUSÃO}

A análise dos setores de fiação e das atividades de trabalho de fiandeiro permitiu identificar fatores de risco físicos e organizacionais reconhecidamente associados aos DORT, bem como uma alta prevalência de dor e/ ou desconforto, a despeito dos investimentos da empresa na inovação tecnológica de um dos setores de fiação. Conclui-se que investimentos em tecnologia que não acompanham mudanças na organização e nas condições de trabalho resultam na manutenção ou no agravamento das situações de risco reconhecidamente associadas aos DORT. 


\section{REFERENNCIAS}

1 Rio RP. LER: ciência e lei. Belo Horizonte: Health; 1998.

2 Santos Filho SB, Barreto SM. Algumas considerações metodológicas sobre os estudos epidemiológicos das lesões por esforços repetitivos (LER). Cad Saude Publ. 1998;14(3):27-32.

3 Sato L. LER: objeto e pretexto para a construção do campo trabalho e saúde. Cad Saude Publ. $2001 ; 17(1): 27-35$.

4 Silverstein BA, Fine LJ, Armstrong TJ. Hand wrist cumulative trauma disorders in industry. $\mathrm{Br} \mathrm{J}$ Ind Med. 1986;43:779-84.

5 Gold JE, Punnett L, Cherniack M, Wegman DH. Digital vibration threshold testing and ergonomic stressors in automobile manufacturing workers: a crosssectional assessment. Ergonomics. 2005;48(1):66-77.

6 Punnett L, Robins JM, Wegman DH, Keyserling WM. Soft tissue disorders in the upper limbs of female garment workers. Scand J Work Environ Health. 1985;11:417-25.

7 Hartmann B, Fleischer AG. Physical load exposure at construction sites. Scand J Work Environ Health. 2005;31(2):88-95.

8 Bjelle A, Hagberg M, Michaelson G. Occupational and individual factors in acute shoulder-neck disorders among industrial workers. Br J Ind Med. 1981;38:356-63.

9 Falck B, Aarnio P. Left-sided carpal tunnel syndrome in butchers. Scand J Work Environ Health. 1985;9:291-7.

10 Ferguson D. An Australian study of telegraphists' cramp. Br J Ind Med. 1971;28:280-5.

11 Ribeiro HP. Lesões por esforços repetitivos (LER): uma doença emblemática. Cad Saude Publ. 1997;13(2):17-24.

12 Chen J-C, Dennerlein JT, Shih T-S, Chen C-J, Cheng Y, Chang WP, et al. Knee pain and driving duration: a secondary analysis of the taxi-drivers health study. Am J Public Health. 2004; 94(4):575-81.

13 National Institute of Occupational, Safety and Health (NIOSH). Musculoskeletal disorders and workplace factors: a critical review of epidemiologic evidence for work-related musculoskeletal disorders of the neck, upper extremity, and low back. Cincinnati $(\mathrm{OH}, \mathrm{USA})$. Department of Health and Human Services, NIOSH; 1997. Disponível em: http://www.cdc.gov/niosh.
14 Chiang H-C, Ko Y-C, Chen S-S, Yu H-S, Wu T-N, Chang P-Y. Prevalence of shoulder and upper-limb disorders among workers in the fish-processing industry. Scand J Work Environ Health. 1993;19:126-3.

15 Tanaka S, Wild DK, Seligman PJ, Halperin WE, Behrens VJ, Putz-Anderson V. Prevalence of workrelatedness of self-reported carpal tunnel syndrome among U.S. workers: analysis of the occupational health supplement data of 1988 National Health Interview Survey. Am J Ind Med. 1995;27:451-70.

16 Silverstein BA, Fine LJ, Armstrong TJ. Occupational factors and carpal tunnel syndrome. Am J Ind Med. 1987; 11:343-58.

17 Brisson C, Vinet A, Vézina M, Gingras S. Effect of duration of employment in piecework on severe disability among female garment workers. Scand J Work Environ Health. 1989;15:329-34.

18 Kerr MS, Frank JW, Shannon HS, Norman RW, Wells RP, Neumann WP, et al. Biomechanical and psychosocial factors for low back pain at work. Am J Public Health. 2001;91(7):1069-75.

19 Huang GD, Feuerstein M, Kop WJ, Schor K, Arroyo F. Individual and combined impacts of biomechanical and work organization factors in work-related musculoskeletal symptoms. Am J Ind Med. 2003; 43(5):495-506.

20 Bongers PM, Kremer AM, ter Laak J. Are psychosocial factors risk factors for symptoms and signs of the shoulder, elbow, or hand/wrist? A review of the epidemiological literature. Am J Ind Med. 2002;41(5):315-42.

21 McAtamney I, Corlett EN. Reducing the risks of work upper limb disorders: a guide and methods. Nothingham: The Institute of Occupational Ergonomics, University of Nothingham; 1992.

22 Kilbom A. Assessment of physical exposure in relation to work-related musculoskeletal disorders: what information can be obtained from systematic observation? Scand J Work Environ Health. 1994;20:30-45.

23 Stock S. Workplace ergonomic factors and the development of musculoskeletal disorders of neck and upper limbs: a meta-analysis. Am J Ind Med. 1991;19:87-107.

24 Burt S, Punnett L. Evaluation of interrater reliability for posture observations in a field study. Appl Ergon. 1999;30:121-35. 


\section{Referências (cont.)}

25 Ranney D, Wells R, Moore A. Upper limb musculoskeletal disorders in highly repetitive industries: precise anatomical physical findings. Ergonomics. 1995;38(7):1408-23.

26 Ranney D. Distúrbios osteomusculares crônicos relacionados ao trabalho. São Paulo: Roca; 2000.

27 Maciel RH. Ergonomia e lesões por esforços repetitivos. In: Codo W, Almeida MCCG, editores. LER: diagnóstico, tratamento e prevenção: uma abordagem interdisciplinar. Petrópolis: Vozes; 1995. p.163-201.

28 Assunção AA, Rocha LE. Agora... até namorar fica difícil: uma história de lesões por esforços repetitivos. In: Bushinelli JT, Rocha LE, Rigotto RM, organizadores. Isto é trabalho de gente? Vida, doença e trabalho no Brasil. São Paulo: Vozes; 1994. p.461-93.

29 Bartys S, Burton K, Main C. A prospective study of psychosocial risk factors and absence due to musculoskeletal disorders: implications for occupational screening. Occup Med. 2005; 55(5):375-9.

30 Abramides MBC, Cabral MSR. Regime de acumulação flexível e saúde do trabalhador. São Paulo Perspec. 2003;17(1):3-10. [periódico on-line]. [citado 29 jan 2007] Disponível em: http://www.seade.gov.br/produtos/ spp/v17n01/v17n01_01.pdf.

31 Dejours C. A loucura do trabalho: estudo de psicopatologia do trabalho. São Paulo: Oboré; 1992.
32 Seligmann-Silva E. Saúde mental e automação: a propósito de um estudo de caso no setor ferroviário. Cad Saude Publ. 1997;13(2):95-109.

33 Queiroz MF, Maciel RH. Condições de trabalho e automação: o caso do soprador da indústria vidreira. Rev Saude Publ. 2001;35(1):1-9.

34 Fassa AG, Faci LA, Dall’Agnol MM. Trabalho e morbidade comum em indústria de celulose e papel: um perfil segundo o setor. Cad Saude Publ. 1996;12(3):297-307.

35 Ghisleni AP, Merlo ARC. Trabalho contemporâneo e patologias por hipersolicitação. Psicol Reflex Crit. 2005;18(2):171-6.

36 Oliveira S. A qualidade da qualidade: uma perspectiva em saúde do trabalhador. Cad Saude Publ. 1997;13(4):625-34.

37 Merlo ARC, Vaz MA, Spode CB, Elbern JLG, Karkow ARM, Vieira PRB. O trabalho entre prazer, sofrimento e adoecimento: a realidade dos portadores de lesões por esforços repetitivos. Psicol Soc. 2003;15(1):117-36.

38 Codo W. Providências na organização do trabalho para a prevenção da LER. In: Codo W, Almeida MCCG, organizadores. LER: diagnóstico, tratamento e prevenção: uma abordagem interdisciplinar. Petrópolis: Vozes; 1998. p.222-48. 